\title{
A new corpus of texture, timbre, and change in 20th-century American popular music
}

\author{
Christopher Wm. White ${ }^{1 \dagger}$, Jeffrey Fulmer ${ }^{2}$, Brian Cordova ${ }^{1}$, Alexandria Black ${ }^{1}$, Chloe Danitz ${ }^{1}$, \\ William Evans ${ }^{1}$, Aidan Fischer ${ }^{1}$, Rashaad Greene, Jinhan He, Emily Kenyon, Joan Miller, \\ Madeline Moylan $^{1}$, Abigail Ring ${ }^{1}$, Emily Schwitzgebel ${ }^{3}$, Yatong Wang ${ }^{1}$ \\ ${ }^{, 1}$ University of Massachusetts Amherst, Amherst, MA, USA \\ ${ }^{2}$ Hampshire College, Amherst, MA, USA \\ ${ }^{3}$ Northwestern University, Evansville, IN, USA \\ $\dagger$ Corresponding author: cwmwhite@umass.edu \\ Published 16 December 2021; https://doi.org/10.18061/FDMC.2021.0015 \\ Author video presentation and/or other conference material: https://doi.org/10.17605/OSF.IO/W32K6
}

\begin{abstract}
This paper describes a new corpus of texture, timbre, and lyrical information within 20th century American popular music, parameters which recent research has suggested play crucial roles in how this music is consumed and perceived. Our corpus applies 3-dimensional annotations to the McGillBillboard corpus (a corpus of harmonic and formal annotations of songs within the Billboard Top-100 between 1958 and 1991; Burgoyne, 2012). These annotations indicate moments of sonic change within each song. The first dimension indicates the broader musical domain of the change (instrumental, percussion, voice, extramusical sounds, lyrics, or rhythm); the second specifies the domain's particular category (e.g.: "backup singers" as a subcategory of the "voice" domain); and, the third indicates whether the texture begins, ends, or alters the previous texture. This paper outlines the methods used to assign these annotations, and some characteristics and notable aspects of the resulting corpus.
\end{abstract}

KEYWORDS: corpus analysis, popular music, lyrics, timbre, texture

\section{Introduction}

To date, corpus analyses of American popular music have primarily focused on the repertoire's use of melody (Tan, Lustig, \& Temperley 2019), harmony (deClercq \& Temperley, 2011; White \& Quinn, 2018), and form (Temperley, 2018). Recent scholarship, however, has suggested that texture and timbre play crucial roles in the production and consumption of American popular music (Barna, 2019; Lavengood, 2017), as well as the repetition of these elements (Acevedo, 2020; Margulis, 2014). This paper outlines annotations added to the McGill-Billboard corpus of $20^{\text {th }}$ century American popular music (Burgoyne, 2012) that indicate moments of instrumental, textural, timbral or other types of sonic changes not explicitly present in the corpus as it currently stands. Our goal is for these annotations to assist research into the interactions between texture, timbre, lyrics and form within this repertoire.

\section{Method}

The McGill-Billboard corpus includes individual text files for each song that specify metadata associated with the song (title, artist, meter, and tonic), and represent each of the song's phrases separate lines, with chords annotated for each measure within the phrases. Microtiming information is associated with each phrase onset, and formal zones (e.g., verse, chorus, intro) are additionally included.

\section{Procedures}

Each of this paper's authors served as annotators. To begin the annotation process, each studied five files along with recordings of the songs, and notated all "moments of change" within the track. As a group, we then compiled a list of all such changes, and both normalized this list using consistent labels, and also decided upon categories with which to organize these changes. Pairs of annotators then independently applied these preliminary annotations to five new songs in the corpus, then compared their results. From our discussions of these comparisons, we developed a style guide, along with a more expansive and rigorous list of possible annotations and categorizations. After repeating this pairwise process a further time, a final list and style guide were decided upon. Using these guides, annotators then progressed through the entire corpus. If a new event type was encountered during this process, a new annotation could be suggested to the collaborators for potential inclusion within the annotation list.

\section{The annotations}

The annotations each have three values, with the first value representing the category of change, the second the genus, and the third the type. Categories involve the broadest band of similarity, and indicate the musical 
domain or instrument group expressing the change. They are: percussion $(\mathrm{P})$, voice $(\mathrm{V})$, extramusical sounds $(\mathrm{E})$, harmony $(\mathrm{H})$, words/lyrics $(\mathrm{W})$, rhythm $(\mathrm{R})$, and instrumental/texture (T). The second value indicates the genera specific to each category. These involve the kind of change being affected (e.g., there is a "solo" genus under the "texture" category, as a solo is a kind of texture). Categories have different numbers of genera, with texture containing the most (12) and rhythm having the fewest (1). The final value then indicates the three event types: Change, Entry, Exit. These types were held in common throughout the categories and genera. The process results in 50 unique three-character annotations, with 14,376 annotations added to 665 McGill-Billboard text files. Figure 1 shows the disposition of annotations between categories.

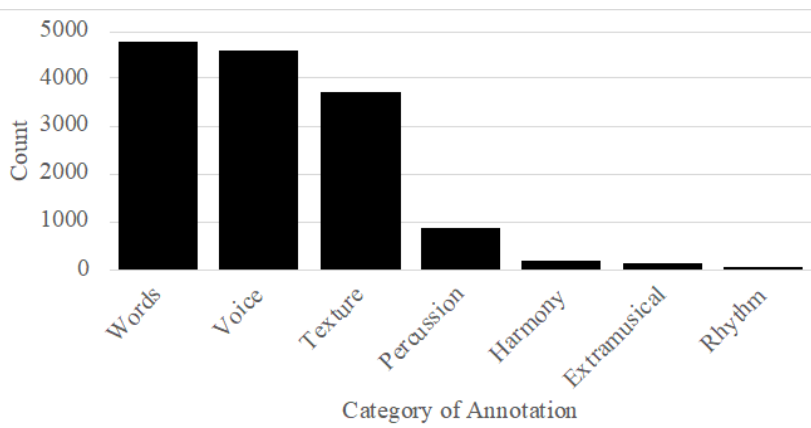

Figure 1: Distribution of categories in the annotation dataset

Each three-member annotation is preceded by a " $\$$ " for querying purposes. Figure 2 shows an example of these annotations from James Brown's "I Don't Mind." Red circles indicate our added annotations, first showing the entry of an accompanying instrument (\$TYI) followed by the entry of backup singers (\$VBI) and a further new singer (\$VSI). A complete dictionary of annotations and the style guide can be found along with the full corpus of annotations at chriswmwhite.com/popannotations.

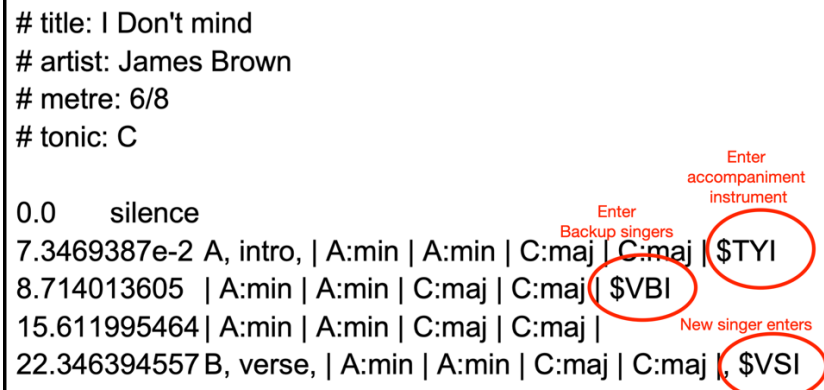

Figure 2: Example annotations

\section{Some Corpus Properties}

Figure 3 displays a series of box-and-whisker plots for each category. The horizontal line within each box shows the median; the upper and lower bounds of the boxes outline the quartiles of data points higher and lower than the median; x's show means; and the range is shown by the "whiskers" emanating from the boxes. This graph illustrates that each category of change can occur throughout a piece, but different categories appear to favor different points within a track's duration. For instance, changes involving percussion seem to occur closer to the beginning of the track, while rhythmic variations occur closer to the end. Figure 4 investigates the disposition of percussion-categorized events, showing each of this category's individual events types: PAI (additional percussion enters, e.g., shaker or wood block); PAO (additional percussion exits); PBI (drums/percussion solo/break starts); $\quad$ PBO (drums/percussion solo/break exits); PTI (full percussion/drum texture enters), PTO (full percussion/drum texture exits). This graph shows that the overall early placement of the percussion category is primarily due to early entries of the full percussion texture and/or additional percussion sounds.

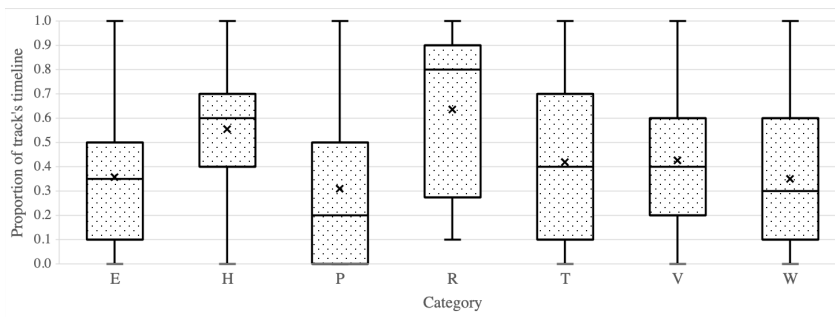

Figure 3: Distribution of categories within pieces' timelines

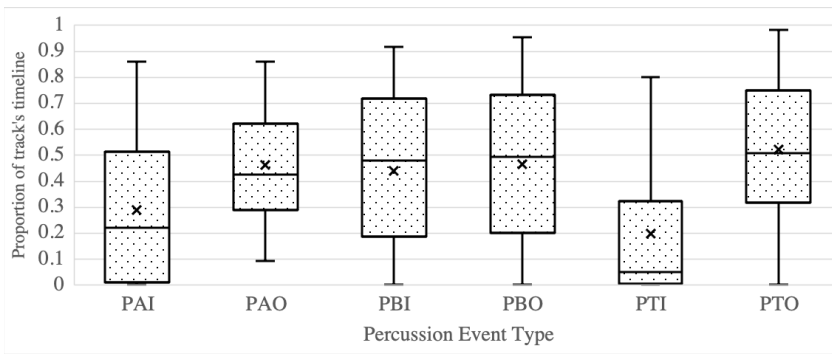

Figure 4: Distribution of events within the Percussion category

Figures 5 and 6 demonstrate some connections between popular music's formal zones and moments of change. Figure 5 shows the average annotations applied to each formal zone in this corpus, with incidental modules (e.g., solos, outros, instrumental interludes) receiving 
the most on average, and choruses and pre-choruses hosting the least. Figure 6 investigates the distribution of these annotations specifically within different rotations of songs' verses. This distribution illustrates that first verses receive the most annotations, while the remainder of verses retain a somewhat steady number of annotations.

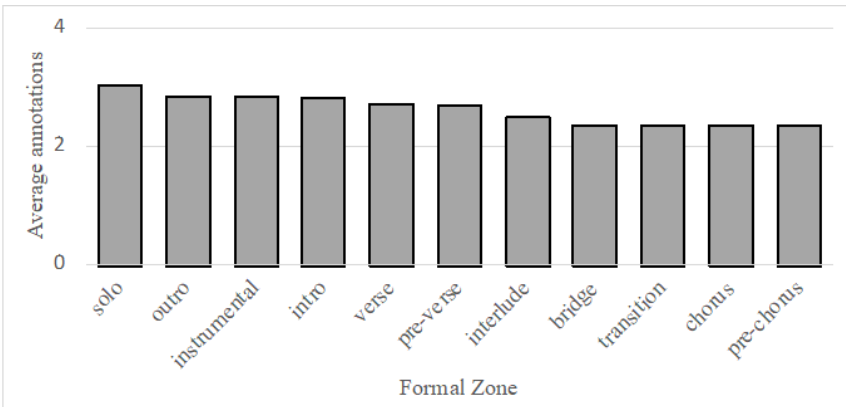

Figure 5: Average amounts of annotations for each formal zone

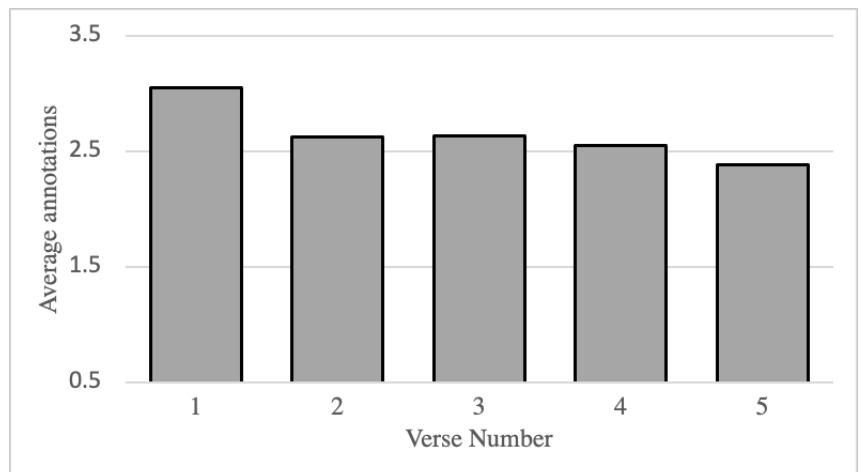

Figure 6: Average amounts of annotations for the first five rotations of verse material

\section{Future Directions}

In an effort to provide maximum coverage of the McGill Billboard Corpus, the final annotations were made by a single collaborator. Individual decisions are likely to vary slightly between annotators, and mistakes and inconsistencies can be expected within the corpus as it currently stands. While we believe the size of the corpus and number of collaborators somewhat mitigate these issues, a second wave of verification and editing would be useful to check for consistency and completeness.

\section{Acknowledgements}

Many thanks to the graduate students of "Analysis of Pop and Rock" at UMass Amherst for participating in this annotation project.

\section{References}

Acevedo, S. (2020). Harmonic schemata of popular music: an empirical representation of analytical patterns and their representation. Ph.D. Dissertation, Yale University.

Barna, A. (2019). Examining contrast in rock and popular music. Ph.D. Dissertation, Eastman University.

Burgoyne, J.A. (2012). Stochastic processes and datasetdriven musicology. Ph.D. dissertation, McGill University.

deClercq, T, \& Temperley, D. (2011). A corpus analysis of rock harmony." Popular Music 30(1), 47-70. https://doi.org/10.1017/S026114301000067X

Lavengood, M. (2017). A new approach to the analysis of musical timbre. Ph.D. diss, CUNY Graduate Center.

Margulis, L. (2014). On repeat: how music plays the mind. New York: Oxford University Press. https://doi.org/10.1093/acprof:oso/9780199990825.001. 0001

Tan, I., Lustig, E., Temperley, D. (2019). Anticipatory syncopation in rock: a corpus study. Music Perception, 36(4), 353-370. https://doi.org/10.1525/mp.2019.36.4.353

Temperley, D. (2018). The musical language of rock. New York: Oxford University Press. https://doi.org/10.1093/oso/9780190653774.001.0001

White, CW. and Quinn, I. (2018). Chord content and harmonic function in tonal music. Music theory spectrum, 40(2), 314-350. https://doi.org/10.1093/mts/mty021 